\title{
Totally Umbilical Lightlike Hypersurfaces in Robertson-Walker Spacetimes
}

\author{
Junhong Dong and Ximin Liu \\ School of Mathematical Sciences, Dalian University of Technology, Dalian 116024, China \\ Correspondence should be addressed to Ximin Liu; ximinliu@dlut.edu.cn
}

Received 25 January 2014; Accepted 24 February 2014; Published 18 March 2014

Academic Editors: T. Friedrich and S. Hervik

Copyright (C) 2014 J. Dong and X. Liu. This is an open access article distributed under the Creative Commons Attribution License, which permits unrestricted use, distribution, and reproduction in any medium, provided the original work is properly cited.

\begin{abstract}
We study the problem of lightlike hypersurface immersed into Robertson-Walker (RW) spacetimes in this paper, where the screen bundle of the hypersurface has constant higher order mean curvature. We consider the following question: under what conditions is the compact lightlike hypersurface totally umbilical? Our approach is based on the relationship between the lightlike hypersurface with its screen bundle and the Minkowski formulae for the screen bundle.
\end{abstract}

\section{Introduction}

The mathematical interests for the study of spacelike hypersurfaces in spacetimes began in the seventies with the works of Cheng and Yau [1], Brill and Flaherty [2], Choquet-Bruhat [3], and later on with some other authors, such as in [4-6]. Moreover, the study of such hypersurfaces is also of interest from a physical point of view, because of its relation to several problems in general relativity. More recently, there has been an increasing interest in the study of spacelike hypersurfaces with constant higher order mean curvature, such as in [79]. At the same time, in $[10,11]$ there are system research works about the lightlike hypersurface of semi-Riemannian manifolds. In this paper, based on the previous result, we want to make an attempt to study the lightlike hypersurfaces of spacetimes. First of all, we are interested in the study of lightlike hypersurfaces in conformally stationary spacetimes, and the screen bundle with constant higher order mean curvature.

First, we use the Newton transformations as the main analytical tool and study the Minkowski-type integral formulas of the lightlike hypersurface. The use of these kinds of formulas in the Lorentzian manifold was first started by Montiel in [12] for the spacelike hypersurface with constant mean curvature in de Sitter spacetimes, and it was continued by Alías et al. [13, 14] for more general spacetimes. Higherorder Minkowski formula for hypersurface was first obtained by Hsiung in [15] in Euclidean space, and by Bivens in [16] in the Euclidean sphere and hyperbolic space. In this paper, we obtain two Minkowski-type integral formulas about the higher-order mean curvature of the lightlike hypersurface as follows, which are called Minkowski formulas I and II.

The Minkowski Formula I. Let $(M, g, S(T M))$ be a compact lightlike hypersurface of a conformally stationary spacetime $\bar{M}$ of constant sectional curvature, on which the screen bundle $S(T M)$ is integrable and Ricci tensor of the induced connection $\nabla$ is symmetric; then the following conclusion holds:

$$
\int_{M}\left(\phi H_{r}+\left\langle\partial_{t}, \xi\right\rangle H_{r+1}^{\prime}+\left\langle\partial_{t}, N\right\rangle H_{r+1}\right) d V=0
$$

The Minkowski Formula II. Let $(M, g, S(T M))$ be a compact lightlike hypersurface immersed into a conformally stationary spacetime $(\bar{M}, g)$ of constant sectional curvature, where the screen bundle $S(T M)$ is integrable and the Ricci tensor of induced connection $\nabla$ is symmetric; then

$$
\int_{M}\left(\phi H_{r}^{\prime}+\left\langle\partial_{t}, \xi\right\rangle H_{r+1}^{\prime}\right) d V=0
$$

Second, by using the Minkowski-type integral formulas and divergence theorem, we get some sufficient conditions for lightlike hypersurfaces of conformally stationary spacetimes which are totally umbilical. The screen bundle of the lightlike hypersurface is spacelike; we study the lightlike hypersurfaces 
of conformally stationary spacetimes via the screen bundle and get our main results (cf. Theorems 10-13).

This paper is organized as follows. In Section 2, we state some preliminary knowledge related to this paper. In Section 3, we introduce the shape operator and the corresponding Newton transformations and derive some formulas about the divergence of Newton transformations. In Section 4, based on the results in Section 3, we obtain the Minkowski-type integral formulas I and II of lightlike hypersurfaces. In Section 5, as the application of Minkowski formulas, we obtain some sufficient conditions for lightlike hypersurfaces totally umbilical when the screen bundle is with constant higher order mean curvature.

\section{Preliminaries}

Let $F^{n+1}$ be an $(n+1)$-dimensional $(n \geq 1)$ Riemannian manifold and let $I$ be a 1-dimensional manifold (either a circle or an open interval of $R$ ). We denote $\bar{M}^{n+2}$ by the $(n+2)$ dimensional product manifold endowed with the Lorentzian metric

$$
\langle,\rangle=-d t^{2}+f^{2}(t)\langle,\rangle_{F},
$$

where $f>0$ is a positive smooth function on $I$, and $\langle,\rangle_{F}$ is the Riemannian metric on $F^{n+1}$; then we have that $\bar{M}$ is a Lorentzian warped product with Lorentzian base $\left(I,-d t^{2}\right)$, Riemannian fiber $F^{n+1},\langle,\rangle_{F}$, and warping function $f$, when the sectional curvature of Riemannian factor $F^{n+1}$ is constant; then we call $-I \times F^{n+1}$ as Robertson-Walker (RW) spacetime.

Consider smooth immersion $\psi: M^{n+1} \hookrightarrow-I \times F^{n+1}$ of an $n+1$-dimensional connected lightlike manifold $M$ into a RW spacetime, where the induced metric on $M$ is $g$; then we have that $M^{n+1}$ is a lightlike hypersurface.

Let $\partial_{t}=\left(\partial / \partial_{t}\right)_{(t, x)},(t, x) \in-I \times F^{n+1}$ be the unitary timelike conformal vector field globally defined on the RW spacetime $\bar{M}$; then consider lightlike hypersurface $(M, g, S(T M))$; there exists a pair lightlike vector fields $\{N, \xi\}$ defined on $M$ which satisfied the following formulas:

$$
\begin{gathered}
\langle\xi, \xi\rangle=\langle N, N\rangle=0, \quad\langle\xi, N\rangle=1, \\
\left\langle\partial_{t}, \xi\right\rangle \neq 0, \quad\left\langle\partial_{t}, N\right\rangle \neq 0 .
\end{gathered}
$$

There exists a unitary timelike vector field $T=N-\xi$ defined on $M$ which is the same time-orientation as $\partial_{t}$; we will refer to $T$ as the future-pointing Gauss map of the hypersurface; its opposite is the past-pointing Gauss map, where we can suppose $\left\langle\xi, \partial_{t}\right\rangle>0$ on $M$ in the future-pointing.

Now we denote $\nabla, \nabla^{*}$ to be the connection of $M$ and $S(T M)$, respectively, $P$ the projection morphism of $T M$ onto $S(T M)$; then the Gauss and Weingarten formula for the hypersurface in $\bar{M}$ are given as follows:

$$
\begin{gathered}
\bar{\nabla}_{X} Y=\nabla_{X} Y+h(X, Y)=\nabla_{X} Y+B(X, Y) N, \\
\bar{\nabla}_{X} N=-A_{N} X+\nabla_{X}^{t} N=-A_{N} X+\tau(X) N, \\
\nabla_{X} P Y=\nabla_{X}^{*} P Y+h^{*}(X, P Y)=\nabla_{X}^{*} P Y+C(X, P Y) \xi, \\
\nabla_{X} \xi=-A_{\xi}^{*} X-\tau(X) \xi, \quad A_{\xi}^{*} \xi=0,
\end{gathered}
$$

where $X, Y \in \Gamma(T M), \xi \in \Gamma(\operatorname{Rad} T M), N \in \Gamma(\operatorname{tr}(T M)), h$ and $h^{*}$ are the second fundamental forms of $M$ and $S(T M)$, $B$ and $C$ are the local second fundamental forms on $\Gamma(T M)$ and $\Gamma(S(T M))$, respectively, $A_{N}$ and $A_{\xi}^{*}$ are the local shape operator on $M$ and $S(T M)$, respectively, and $\tau$ is the 1-form on TM.

Definition 1. $(M, g, S(T M))$ is a lightlike hypersurface of a semi-Riemannian manifold $(\bar{M}, \bar{g})$. A point $u \in M$ is said to be umbilical if

$$
B_{u}\left(X_{u}, Y_{u}\right)=k g_{u}\left(X_{u}, Y_{u}\right), \quad X_{u}, Y_{u} \in T_{u} M,
$$

where $B$ is the local second fundamental form of $M$ defined around $u$ and $k \in R$. One says $M$ is totally umbilical if any point of $M$ is umbilical.

Then it is easy to see that $M$ is totally umbilical if and only if on $M$ there exists a smooth function $\rho$ on $M$ such that

$$
B(X, Y)=\rho g(X, Y),
$$

for all vector fields $X, Y \in \Gamma(T M)$.

Definition 2. Let $(M, g, S(T M))$ be a lightlike hypersurface; then the screen bundle is totally umbilical if on any coordinate neighbourhood $U \subset M$ there exist a smooth function $\lambda$ such that

$$
C(X, P Y)=\lambda g(X, P Y), \quad X, Y \in \Gamma(T M) .
$$

In case $\lambda=0(\lambda \neq 0)$ on $U$ one says $S(T M)$ is totally geodesic (properly totally umbilical).

\section{The Shape Operator and Newton Transformation}

In this section we will introduce the shape operators and the corresponding Newton transformations $T_{r}$.

From [10] we have the relationship between two local second fundamental forms with the shape operators

$$
\begin{gathered}
B(X, Y)=\left\langle A_{\xi}^{*} X, Y\right\rangle, \quad C(X, P Y)=\left\langle A_{N} X, P Y\right\rangle, \\
B(X, \xi)=\left\langle A_{\xi}^{*} X, N\right\rangle=0, \quad\left\langle A_{N} X, N\right\rangle=0,
\end{gathered}
$$

where $X, Y \in \Gamma(T M), \xi \in \Gamma\left(T M^{\perp}\right)$, and $N \in \Gamma(\operatorname{tr} T M)$. From (11) we have that $A_{N} X$ belong to screen bundle $S(T M)$, if $A_{N} \xi=0$ and $S(T M)$ is an integrable distribution; then it is easy to know $A_{N}$ is self-adjoint linear operator in each tangent space $T_{p} M$; its eigenvalues $k_{1}, k_{2}, \ldots, k_{n}$ and $k_{n+1}$ are the principal curvature of the lightlike hypersurface. If $\left\{e_{1}, \ldots, e_{n}, \xi\right\}$ are the eigenvector of $A_{N}$ corresponding to the eigenvalue $k_{1}(p), k_{2}(p), \ldots, k_{n}(p)$ and $k_{n+1}(p)$ on $T_{p} M$, respectively, where $A_{N}\left(e_{i}\right)_{p}=k_{i}(p) e_{i}, 1 \leq i \leq n$ and $k_{n+1}=0$, then the matrix of $A_{N}$ with respect to the orthogonal basis $\left\{e_{1}, \ldots, e_{n}, \xi\right\}$ is given by

$$
\left(\begin{array}{cccc}
k_{1} & \cdots & 0 & 0 \\
\vdots & \ddots & \vdots & \vdots \\
0 & \cdots & k_{n} & 0 \\
0 & \cdots & 0 & 0
\end{array}\right)
$$


Now we restrict $A_{N}$ on $S(T M)$; then it can be denoted by

$$
\left(\begin{array}{ccc}
k_{1} & \cdots & 0 \\
\vdots & \ddots & \vdots \\
0 & \cdots & k_{n}
\end{array}\right) .
$$

Proposition 3. Let $(M, g, S(T M))$ be a lightlike hypersurface of $(\bar{M}, \bar{g})$; then the screen bundle is totally umbilical if and only if there exist a smooth function $\lambda$ such that

$$
A_{N} X=\lambda X, \quad A_{N} \xi=0, \quad X \in \Gamma(S(T M)) .
$$

Proposition 4 (see [10]). $\left(M^{n+1}, g, S(T M)\right)$ is a lightlike hypersurface of a semi-Riemannian manifold $(\bar{M}, \bar{g})$ of constant sectional curvature $c$ and totally umbilical screen bundle $S(T M)$; that is, there exists a smooth function $\lambda$ making $A_{N} X=\lambda X$ and $A_{N} \xi=0$. Then $M$ is totally umbilical and immersed into $\bar{M}$ if $\lambda \neq 0$. Under this condition, we say $M$ is totally geodesic immersed into $\bar{M}$ if and only if $\lambda$ is a solution of the partial differential equation $\xi(\lambda)-\lambda \tau(\xi)+c=0$.

The proof of Proposition 4 has been given by Duggal and Bejancu in [10].

From Proposition 4 and the characters of totally umbilical lightlike hypersurface, we can obtain the following corollary.

Corollary 5. Let $(M, g, S(T M))$ be a lightlike hypersurface of $(\bar{M}(c), \bar{g})$, where the sectional curvature of ambient space $\bar{M}$ is constant $c$. Now one supposes the screen bundle $S(T M)$ is integrable; $A_{N} \xi=0$ and $A_{N} X=\lambda X(\lambda \neq 0)$, where $\lambda$ is a smooth function. Then $M$ is totally umbilical; that is, $A_{\xi} Y=$ $\rho Y$, where $X, Y \in \Gamma(S(T M))$. Besides $\rho, \lambda$, and $c$ satisfied the following equation:

$$
\xi(\lambda)-\lambda \tau(\xi)+c=\rho \lambda, \quad \xi \in \Gamma\left(T M^{\perp}\right) .
$$

From Corollary 5 we know that $M^{n+1}$ is a totally umbilical hypersurface in $\bar{M}(c)$; if there exists a smooth function $\lambda$ such that $A_{N}=\lambda I$ on screen bundle and $A_{N} \xi=0$, where $I$ denotes the identity in $S(T M)$.

Associated with the shape operator $A_{N}$ there are $n$ algebraic invariants given by $S_{r}$ as follows:

$$
S_{r}(p)=\sigma_{r}\left(k_{1}(p), k_{2}(p), \ldots, k_{n}(p)\right), \quad 1 \leq r \leq n,
$$

where $\sigma: R^{n} \rightarrow R$ are the elementary symmetric functions in $R^{n}$ given by

$$
\sigma_{r}\left(x_{1}, x_{2}, \ldots, x_{n}\right)=\sum_{i_{1}<\cdots<i_{r}} x_{i_{1}} \cdots x_{i_{r}} .
$$

Then the characteristic polynomial of $A_{N}$ restrict on $S(T M)$ can be denoted by

$$
\operatorname{det}\left(t I-A_{N}\right)=\sum_{r=0}^{n}(-1)^{r} S_{r} t^{n-r},
$$

where $S_{0}=1$. Now we denote $H_{r}$ by $\left(\begin{array}{c}n \\ r\end{array}\right) H_{r}=(-1)^{r} S_{r} ; H_{r}$ is the $r$-mean curvature of the screen bundle with respect to the shape operator $A_{N}$. In particular, when $r=1, H_{1}=$ $(1 / n) \operatorname{tr}\left(A_{N}\right)$ is the main extrinsic mean curvature of the screen bundle with respect to $A_{N}$; when $r=2, H_{2}$ defines a 2mean curvature which is intrinsic. Thus, if all $k_{i}$ are nonzero, we can choose a proper orientation to make $H_{1}>0$ and $H_{2}$ unchanged.

The curvature tensor $\bar{R}$ of the ambient space $\bar{M}$ can be written as follows:

$$
\begin{aligned}
& \bar{R}(X, Y) Z= R(X, Y) Z+A_{h(X, Z)} Y-A_{h(Y, Z)} X \\
&+\left(\nabla_{X} h\right)(Y, Z)-\left(\nabla_{Y} h\right)(X, Z), \\
& R(X, Y) Z=(\bar{R}(X, Y) Z)^{T}-A_{h(X, Z)} Y+A_{h(Y, Z)} X,
\end{aligned}
$$

where $\left(\nabla_{X} h\right)(Y, Z)=\nabla_{X}^{t}(h(Y, Z))-h\left(\nabla_{X} Y, Z\right)-$ $h\left(Y, \nabla_{X} Z\right),(\bar{R}(X, Y) Z)^{T}$ is the tangential component of $\bar{R}(X, Y) Z$.

Now we denote $\left\{E_{0}, E_{i}, N\right\}$ to be the basis on $M$ and Ric the Ricci tensor of $M$, where $1 \leq i \leq n$; that is, $\xi=E_{0},\left\{E_{i}\right\}$ the basis of $S(T M)$; then from [10] we can obtain that

$$
\begin{aligned}
\operatorname{Ric}\left(E_{i}, E_{j}\right)= & \overline{\operatorname{Ric}}\left(E_{i}, E_{j}\right)-\bar{g}\left(\bar{R}\left(E_{i}, E_{0}\right) E_{j}, N\right) \\
& -g^{k t}\left(C\left(E_{k}, E_{t}\right) B\left(E_{i}, E_{j}\right)\right. \\
& \left.-C\left(E_{i}, E_{t}\right) B\left(E_{j}, E_{k}\right)\right) .
\end{aligned}
$$

So we obtain

$$
\begin{aligned}
\operatorname{Ric}( & \left.E_{i}, E_{j}\right)-\operatorname{Ric}\left(E_{j}, E_{i}\right) \\
= & g^{k t}\left\{C\left(E_{i}, E_{t}\right) B\left(E_{j}, E_{k}\right)-C\left(E_{j}, E_{t}\right) B\left(E_{i}, E_{k}\right)\right\} \\
& +\bar{g}\left(\bar{R}\left(E_{j}, E_{i}\right) E_{0}, N\right) \\
= & A_{j}^{k} B_{k i}-A_{i}^{k} B_{k j}+\bar{R}_{0 i j}^{0} \\
= & 2 d \tau\left(\frac{\partial}{\partial u_{k}}, \frac{\partial}{\partial u_{h}}\right),
\end{aligned}
$$

where $1 \leq k, t \leq n, \tau$ is the 1 -form induced by $S(T M)$. When $i=0$ we have

$$
\operatorname{Ric}\left(E_{0}, E_{j}\right)-\operatorname{Ric}\left(E_{j}, E_{0}\right)=-A_{0}^{k} B_{k j}+\bar{R}_{00 k}^{0}=2 d \tau\left(E_{0}, E_{j}\right) .
$$

By using the equations above we obtain that $d \tau=0$ when the Ricci tensor Ric is symmetric; then we have the following proposition.

Proposition 6. Let $(M, g, S(T M))$ be a lightlike hypersurface of a RW spacetime which is equipped with a conformal vector field $\partial_{t}$. If the Ricci tensor of the induced connection $\nabla$ is symmetric, then there exists a pair $\{\xi, N\}$ on $U$ such that the corresponding 1-form $\tau$ vanishes on any $U \subset M$; besides $\left\langle\partial_{t}, \xi\right\rangle$ and $\left\langle\partial_{t}, N\right\rangle$ are all nonzero.

Proof. It is already known that there exist vector fields $\xi$ and $N$ such that $\left\langle\partial_{t}, \xi\right\rangle \neq 0,\left\langle\partial_{t}, N\right\rangle \neq 0$. 
Since the Ricci tensor of $\nabla$ is symmetric, then we have that the 1 -form $\tau$ induced by $S(T M)$ is closed; that is, $d \tau \equiv 0$; so we have $\tau=\alpha$.

If $\tau=\alpha=0$, then the theorem have been proved.

If $\tau=\alpha \neq 0$, we take $\bar{\xi}=\alpha \xi, \bar{N}=(1 / \alpha) N$, the corresponding 1 -form is $\bar{\tau}$, where

$$
\begin{gathered}
\bar{\tau}(X)=\bar{g}\left(\nabla_{X}^{t} \bar{N}, \bar{\xi}\right)=\alpha X\left(\frac{1}{\alpha}\right)+\tau(X), \\
\tau(X)=\bar{\tau}(X)-\alpha X\left(\frac{1}{\alpha}\right)=\bar{\tau}(X)-X(\log \alpha) .
\end{gathered}
$$

By using Poincaré lemma we obtain $\tau(X)=X(f)$, where $f \in C^{\infty}(U)$. Then we can let $\alpha=e^{f}$, so $\bar{\tau}(X)=0$ for any $X \in \Gamma\left(T M_{U}\right)$. Since $\left\langle\partial_{t}, \bar{\xi}\right\rangle=\alpha\left\langle\partial_{t}, \xi\right\rangle \neq 0,\left\langle\partial_{t}, \bar{N}\right\rangle=$ $(1 / \alpha)\left\langle\partial_{t}, N\right\rangle \neq 0$, then the $\{\bar{\xi}, \bar{N}\}$ is the vector fields satisfying the proposition.

From Proposition 6 we obtain that if the Ricci tensor of $\nabla$ is symmetric, then we have

$$
\begin{aligned}
\langle\bar{R}(X, Y) P Z, N\rangle & =\left\langle\left(\nabla_{X} C\right)(Y, P Z)-\left(\nabla_{Y} C\right)(X, P Z), \xi\right\rangle \\
& =\left\langle\left(\nabla_{X} A_{N}\right) Y-\left(\nabla_{Y} A_{N}\right) X, P Z\right\rangle
\end{aligned}
$$

where $\left(\nabla_{X} C\right)(Y, P Z)=X(C(Y, P Z))-C\left(\nabla_{X} Y, P Z\right)-$ $C\left(Y, \nabla_{X}^{*} P Z\right), \nabla_{X} A_{N}$ is the covariant derivative of $A_{N}$.

Because the ambient space is RW spacetime, the sectional curvature is constant; then when $X, Y \in \Gamma(S(T M))$, we have that $\bar{R}(X, Y) Z=c\{g(Y, Z) X-g(X, Z) Y\}$ has no component in $\Gamma\left(T M^{\perp}\right)$. Now $(24)$ becomes

$$
\left(\nabla_{X} A_{N}\right) Y=\left(\nabla_{Y} A_{N}\right) X
$$

Now we introduce the Newton transformations

$$
T_{r}: \Gamma(S(T M)) \longrightarrow \Gamma(S(T M)), \quad 0 \leq r \leq n,
$$

which arises from the shape operator $A_{N}$. According to the definition of the $r$-mean curvature of screen bundle $S(T M)$, the Newton transformations are given by

$$
T_{r}=\left(\begin{array}{c}
n \\
r
\end{array}\right) H_{r} I+\left(\begin{array}{c}
n \\
r-1
\end{array}\right) H_{r-1} A_{N}+\cdots+\left(\begin{array}{c}
n \\
1
\end{array}\right) H_{1} A_{N}^{r-1}+A_{N}^{r},
$$

where $I$ denotes the identify in $\Gamma(S(T M)), 1 \leq r \leq n$ and $T_{0}=I$. Besides,

$$
T_{r}=\left(\begin{array}{c}
n \\
r
\end{array}\right) H_{r} I+A_{N} T_{r-1}
$$

According to the Cayley-Hamilton theorem, we have $T_{n}=0$. When $r$ is even, the definition of $T_{r}$ does not depend on the chosen Gauss map; when $k$ is odd there is a change of sign in the definition of $T_{r}$.

It is easy to know that $T_{r}(p)$ is also a self-adjoint linear operator with respect to $A_{N}(p)$; besides, $A_{N}(p)$ and
$T_{r}(p)$ can be a simultaneous diagonalization. If $\left\{e_{1}, \ldots, e_{n}\right\}$ is the orthogonal frame on $S(T M)$ which diagonalizes $A_{N_{p}}$, $A_{N_{p}}\left(e_{i}\right)=k_{i}(p) e_{i}, T_{r}$ are all self-adjoint operators with respect to $A_{N}$, then we have

$$
T_{r}\left(e_{i}\right)=\lambda_{i, r}\left(e_{i}\right) \text {, }
$$

where

$$
\lambda_{i, r}=\sum_{i_{1}<\cdots<i_{r}, i_{j} \neq i} k_{i_{1}} \cdots k_{i_{r}}
$$

Then we obtain

$$
\begin{gathered}
\operatorname{tr}\left(T_{r}\right)=c_{r} H_{r}, \\
\operatorname{tr}\left(A_{N} \circ T_{r}\right)=-c_{r} H_{r+1},
\end{gathered}
$$

where $c_{r}=(n-r)\left(\begin{array}{l}n \\ r\end{array}\right)$.

The divergence of $T_{r}$ on screen bundle is defined by

$$
\operatorname{div}_{S(T M)} T_{r}=\operatorname{tr}\left(\nabla T_{r}\right)=\sum_{i=1}^{n}\left(\nabla_{e_{i}} T_{r}, e_{i}\right)
$$

Proposition 7. Let $(M, g, S(T M))$ be a lightlike hypersurface of a RW spacetime in which the screen bundle $S(T M)$ is integrable and Ricci tensor of the induced connection $\nabla$ is symmetric; when the sectional curvature of the ambient spacetime is constant, then the divergence of Newton transformations $T_{r}$ is given by the following inductive formula:

$$
\operatorname{div}_{S(T M)} T_{0}=0
$$

$$
\operatorname{div}_{S(T M)} T_{r}=A_{N}\left(\operatorname{div} T_{r-1}\right)+\sum_{i=1}^{n}\left(\bar{R}\left(N, T_{r-1} e_{i}\right) e_{i}\right)^{*},
$$

where $(\bar{R}(N, X) Y)^{*}$ denotes the screen bundle component of $\bar{R}(N, X) Y$. Besides, for any vector field $X \in \Gamma(S(T M))$ it satisfies

$$
\left\langle\operatorname{div}_{S(T M)} T_{r}, X\right\rangle=\sum_{j=1}^{r} \sum_{i=1}^{n}(-1)^{j}\left\langle\bar{R}\left(\xi, T_{r-j} e_{i}\right) e_{i}, A_{\xi}^{j-1} X\right\rangle .
$$

Proof. It is easy to know $\operatorname{div}_{S(T M)} T_{0}=\operatorname{div} I=0$, when $r \geq 1$, $X, Y \in \Gamma(S(T M))$; we have

$$
\begin{aligned}
& \left(\nabla_{X} T_{r}\right) Y \\
& \quad=\left(\begin{array}{c}
n \\
r
\end{array}\right)\left\langle\nabla H_{r}, X\right\rangle Y+\nabla_{X}\left(A_{N} T_{r-1}\right) Y \\
& \quad=\left(\begin{array}{c}
n \\
r
\end{array}\right)\left\langle\nabla H_{r}, X\right\rangle Y+\left(\nabla_{X} A_{N}\right) T_{r-1} Y+A_{N}\left(\left(\nabla_{X} T_{r-1}\right) Y\right) .
\end{aligned}
$$


Then

$$
\begin{aligned}
& \operatorname{div}_{S(T M)} T_{r} \\
& =\sum_{i=1}^{n}\left(\nabla_{e_{i}} T_{r}\right)\left(e_{i}\right) \\
& =\left(\begin{array}{c}
n \\
r
\end{array}\right) \nabla H_{r}+\sum_{i=1}^{n}\left(\nabla_{e_{i}} A_{N}\right)\left(T_{r-1} e_{i}\right)+A_{N}\left(\operatorname{div}_{S(T M)} T_{r-1}\right) .
\end{aligned}
$$

Using (24) we get for any vector field $X \in \Gamma(S(T M))$; we have

$$
\begin{aligned}
\left\langle\left(\nabla_{e_{i}} A_{N}\right)\left(T_{r-1} e_{i}\right), X\right\rangle= & \left\langle\left(\nabla_{X} A_{N}\right) e_{i}, T_{r-1} e_{i}\right\rangle \\
& +\left\langle\bar{R}\left(e_{i}, X\right) T_{r-1} e_{i}, N\right\rangle \\
= & \left\langle T_{r-1}\left(\left(\nabla_{e_{i}} A_{N}\right) e_{i}\right), e_{i}\right\rangle \\
& -\left\langle\bar{R}\left(N, T_{r-1} e_{i}\right) e_{i}, X\right\rangle . \\
\left\langle\operatorname{div}_{S(T M)} T_{r}, X\right\rangle= & \left(\begin{array}{c}
n \\
r
\end{array}\right)\left\langle\nabla H_{r}, X\right\rangle+\operatorname{tr}\left(T_{r-1} \nabla_{X} A_{N}\right) \\
& -\sum_{i=1}^{n}\left\langle\bar{R}\left(N, T_{r-1} e_{i}\right) e_{i}, X\right\rangle \\
& +\left\langle A_{N}\left(\operatorname{div}_{S(T M)} T_{r-1}\right), X\right\rangle .
\end{aligned}
$$

By using equation (4.4) in [7], we have

$$
\operatorname{tr}\left(T_{r-1} \nabla_{X} A_{N}\right)=\left(\begin{array}{l}
n \\
r
\end{array}\right)\left\langle\nabla H_{r}, X\right\rangle .
$$

From (24) and the above formula, we have

$$
\begin{aligned}
& \left\langle\operatorname{div}_{S(T M)} T_{r}, X\right\rangle \\
& =-\left\langle\bar{R}\left(N, T_{r-1} e_{i}\right) e_{i}, X\right\rangle+\left\langle A_{N}\left(\operatorname{div}_{S(T M)} T_{r-1}\right), X\right\rangle \\
& =\left\langle-\bar{R}\left(N, T_{r-1} e_{i}\right) e_{i}+A_{N}\left(\operatorname{div}_{S(T M)} T_{r-1}\right), X\right\rangle \\
& =-\sum_{j=1}^{r} \sum_{i=1}^{n}\left\langle\bar{R}\left(N, T_{r-j} e_{i}\right) e_{i}, A_{N}^{j-1} X\right\rangle .
\end{aligned}
$$

Since the sectional curvature of the RW spacetime is constant, then it is easy to know $\langle\bar{R}(N, X) Y, Z\rangle=$ $\langle\bar{R}(N, X) Y, Z\rangle=0$, for all vector fields $X, Y, Z \in \Gamma(S(T M))$. From (25) and (34) we have $\operatorname{div}_{S(T M)} T_{r}=0,0 \leq r \leq n$; thus we have the corollary below.

Corollary 8. Let $(M, g, S(T M))$ be a lightlike hypersurface of a conformally stationary spacetime in which the screen bundle $S(T M)$ is integrable and Ricci tensor of the induced connection $\nabla$ is symmetric; then we have that the Newton transformations on $S(T M)$ are divergence-free: $\operatorname{div}_{S(T M)} T_{r}=0,0 \leq r \leq n$.

\section{Minkowski Integral Formula for Lightlike Hypersurface in RW Spacetime}

In this section, by using divergence theorem we derive some general integral formulae for compact lightlike hypersurface in a RW spacetime $\bar{M}$. In order to get that, we consider the vector field $K(t, x)=f(t)(\partial / \partial t)_{(t, x)},(t, x) \in-I \times{ }_{f} F^{n+1}$; it determines a nonvanishing future-pointing closed conformal vector field on $-I \times{ }_{f} F^{n+1}$; besides,

$$
\bar{\nabla}_{X} K=f^{\prime}(t) X
$$

for any vector field $X$ tangent to $-I \times{ }_{f} F^{n+1}$ at a point $(t, x)$.

We denote the height function of $M$ by $h$, which is the restriction of the projection $\pi_{I}(t, x)=t$ to $M ; h: M \rightarrow I$ is given by $h=\pi_{I} \circ \psi$. Observe the gradient of $\pi_{I}$ on $-I \times_{f} F^{n+1}$ is given by

$$
\bar{\nabla} \pi_{I}=-\left\langle\bar{\nabla} \pi_{I}, \partial_{t}\right\rangle \partial_{t}=-\partial_{t}
$$

then we can get the gradient of $h$ on $M$ is

$$
\nabla h=\left(\bar{\nabla} \pi_{I}\right)^{T}=-\partial_{t}^{T},
$$

where $\partial_{t}^{T}=\partial_{t}-\left\langle\xi \partial_{t},\right\rangle N, \partial_{t}^{T}$ is the tangential component of $\partial_{t}$. Now we denote $g: I \rightarrow R$ is an arbitrary primitive of $f$; that is, $g^{\prime}=f>0$; so we can consider $g$ as a reparametrization of the height function. Then we have

$$
\nabla g(h)=f(h) \nabla h=-f(h) \partial_{t}^{T}=-K^{T}
$$

where $K^{T}=K-\langle K, \xi\rangle N$. From (41) we get

$$
\bar{\nabla}_{X} K=f^{\prime}(h) X,
$$

for any $X \in \Gamma(T M)$.

Since $\nabla_{X} K^{T}=f^{\prime}(h) X+\langle K, \xi\rangle A_{N} X$ for every $X \in \Gamma(T M)$, then we have

$$
\nabla_{X}(\nabla h)=-\nabla_{X} K^{T}=-f^{\prime}(h) X-\langle K, \xi\rangle A_{N} X
$$

From $\nabla h=(1 / f(h)) \nabla g(h)$ we have

$$
\begin{aligned}
\nabla_{X}(\nabla h) & =\nabla_{X}\left(\frac{1}{f(h)} \nabla g(h)\right) \\
& =-\frac{f^{\prime}(h)}{f(h)} \nabla h+\frac{1}{f(h)}\left(-f^{\prime}(h) X-\langle K, \xi\rangle A_{N} X\right) \\
& =-(\log f)^{\prime}(h)(\langle\nabla h, X\rangle \nabla h+X)-\left\langle\partial_{t}, \xi\right\rangle A_{N} X
\end{aligned}
$$

If we take $K^{T}$ as the tangential component of $K$ along the hypersurface, we have

$$
K^{T}=K-\langle K, \xi\rangle N
$$


where $\xi \in \Gamma\left(T M^{\perp}\right)$ and $\langle\xi, N\rangle=1$; then we have

$$
\begin{aligned}
\nabla_{X} K^{T}= & \bar{\nabla}_{X}(K-\langle K, \xi\rangle N)-B\left(X, K^{T}\right) N \\
= & f^{\prime}(h) X-\bar{\nabla}_{X}(\langle K, \xi\rangle N)-B\left(X, K^{T}\right) N \\
= & f^{\prime}(h) X+\langle K, \xi\rangle A_{N} X+\left\langle K, A_{\xi}^{*} X\right\rangle N \\
& -\left\langle K^{T}, A_{\xi}^{*} X\right\rangle N \\
= & f^{\prime}(h) X+\langle K, \xi\rangle A_{N} X .
\end{aligned}
$$

Now we have

$$
\begin{aligned}
\nabla_{e_{i}} K^{T} & =\left\langle f^{\prime}(h) e_{i}+\langle K, \xi\rangle A_{N} e_{i}, T_{r} e_{i}\right\rangle \\
& =f^{\prime}(h)\left\langle e_{i}, T_{r} e_{i}\right\rangle+\langle K, \xi\rangle\left\langle A_{N} T_{r} e_{i}, e_{i}\right\rangle .
\end{aligned}
$$

In order to compute the divergence of $K^{T}$, we have to expend the definition of operator $T_{r}$ to the tangential bundle. Now let $A_{N} \xi=0$ and $\left\{e_{1}, \ldots, e_{n}, \xi\right\}$ be the local orthogonal frame on $M$; then $A_{N}$ with respect to the basis can be denoted by

$$
\left(\begin{array}{cccc}
k_{1} & \cdots & 0 & 0 \\
\vdots & \ddots & \vdots & \vdots \\
0 & \cdots & k_{n} & 0 \\
0 & \cdots & 0 & 0
\end{array}\right)
$$

in this case, we denote it by $\bar{A}_{N}$ to distinguish it from $A_{N}$ restricted on $S(T M)$. By a direct calculation we obtain

$$
\operatorname{det}\left(t I-\bar{A}_{N}\right)=t \cdot \operatorname{det}\left(t I-A_{N}\right)=\sum_{r=0}^{n}\left(\begin{array}{l}
n \\
r
\end{array}\right) T_{r} t^{n-r+1}
$$

Then $\bar{T}_{r}=\left(\begin{array}{cc}T_{r} & 0 \\ 0 & \left.\begin{array}{c}n \\ r\end{array}\right) H_{r}\end{array}\right), \operatorname{div}_{M} \bar{T}_{r}=\operatorname{div}_{M}\left(\begin{array}{l}n \\ r\end{array}\right) H_{r} \cdot \operatorname{div}_{M} T_{r}$, $\bar{T}_{r} e_{i}=\lambda_{i, r} e_{i}$. From all the above, we have the theorem below.

Theorem 9. Let $\left(M^{n+1}, g, S(T M)\right)$ be a compact lightlike hypersurface immersed into a $R W$ spacetime $(\bar{M}, g)$, where the screen bundle $S(T M)$ is integrable, $A_{N} \xi=0$, and the Ricci tensor of induced connection $\nabla$ is symmetric. Then the following formula holds:

$$
\begin{aligned}
\int_{M} & \operatorname{div}_{M}\left(\overline{T_{r}} K^{T}\right) d V \\
& =\int_{M} c_{r}\left(f^{\prime}(h) H_{r}-\langle K, \xi\rangle H_{r+1}\right) d V=0 .
\end{aligned}
$$

Proof. Since the sectional curvature of RW spacetime is constant, then we have the calculation about the divergence of $\overline{T_{r}} K^{T}$ as follows:

$$
\begin{aligned}
\operatorname{div}_{M}\left(\bar{T}_{r} K^{T}\right)= & \operatorname{div}_{S(T M)}\left(\bar{T}_{r} K^{T}\right)+\langle\xi, \xi\rangle\left\langle\bar{\nabla}_{\xi} \bar{T}_{r} K^{T}, \xi\right\rangle \\
= & \operatorname{div}_{S(T M)}\left(\bar{T}_{r} K^{T}\right) \\
= & \left\langle\operatorname{div}_{S(T M)} \bar{T}_{r}, K^{T}\right\rangle+\sum_{i=1}^{n}\left\langle\nabla_{e_{i}} K^{T}, \bar{T}_{r} e_{i}\right\rangle \\
= & \left\langle\operatorname{div}_{S(T M)} T_{r}^{\prime} \cdot \operatorname{div}_{S(T M)}\left(\begin{array}{l}
n \\
r
\end{array}\right) H_{r}, K^{T}\right\rangle \\
& +f^{\prime}(h) \operatorname{tr}\left(T_{r}\right)+\langle K, \xi\rangle \operatorname{tr}\left(A_{N} T_{r}\right) \\
= & \left\langle\operatorname{div}_{S(T M)} T_{r} \cdot \operatorname{div}_{S(T M)}\left(\begin{array}{l}
n \\
r
\end{array}\right) H_{r}, K^{T}\right\rangle \\
& +c_{r}\left(f^{\prime}(h) H_{r}-\langle K, \xi\rangle H_{r+1}\right) .
\end{aligned}
$$

Since the sectional curvature of RW spacetime is constant, by using Proposition 7 we have $\operatorname{div}_{S(T M)} T_{r}=0$; then

$$
\operatorname{div}_{M}\left(\overline{T_{r}} K^{T}\right)=c_{r}\left(f^{\prime}(h) H_{r}-\langle K, \xi\rangle H_{r+1}\right) .
$$

From divergence theorem we have

$$
\int_{M}\left(f^{\prime}(h) H_{r}-\langle K, \xi\rangle H_{r+1}\right) d V=0 .
$$

We call the formula (53) as Minkowski-type integral formula.

\section{Umbility of the Compact Lightlike Hypersurface in RW Spacetime}

In this section, we will derive some application of Minkowski formulae for the case of lightlike hypersurface immersed into a spatially closed RW spacetime, where the screen bundle of the hypersurface has constant $r$-mean curvature; under this condition by using (35), it is easy to know that if $M$ is totally umbilical, then $H_{r}$ must be constant.

Theorem 10. Let $\bar{M}=-I \times{ }_{f} F^{n+1}$ be spatially closed $R W$ spacetime; then every compact lightlike hypersurface immersed into $\bar{M}$, where $A_{N} \xi=0$, screen bundle $S(T M)$ is integrable and Ricci tensor of the induced connection is symmetric. If $H_{1}$ of the screen bundle with respect to $A_{N}$ is constant, then the hypersurface must be totally umbilical.

Proof. Using the Theorem 9, It is easy to see that if $H_{1}^{\prime}$ is constant, we can consider $r=0$, then multiplying the Minkowski-type formula by $H_{1}$ we have

$$
\int_{M}\left(f^{\prime}(h) H_{1}-\langle K, \xi\rangle H_{1}^{2}\right) d V=0 .
$$


When $r=1$, we obtain that the Minkowski formula is

$$
\int_{M}\left(f^{\prime}(h) H_{1}+\langle K, \xi\rangle H_{2}\right) d V=0 .
$$

Subtracting the two formulas above we get

$$
\int_{M}\langle K, \xi\rangle\left(H_{1}^{2}-H_{2}\right) d V=0
$$

from the Cauchy-Schwarz inequality we have

$$
H_{1}^{2}-H_{2}=\frac{1}{n(n-1)}\left(\sum_{i=1}^{n} k_{i}^{2}-\frac{1}{n}\left(\sum_{i=1}^{n} k_{i}\right)^{2}\right) \geq 0
$$

where the equality holds only at the point $k_{1}=k_{2}=\cdots=$ $k_{n}$. From $\langle K, \xi\rangle>0$ we have that the equality holds; then there exists constant $\lambda$ such that $A_{N}=\lambda I$ on screen bundle. Besides, $A_{N} \xi=0$; then by using Corollary 5 we have that the hypersurface is totally umbilical.

Theorem 11. Let $\bar{M}=-I \times{ }_{f} F^{n+1}$ be a spatially closed $R W$ spacetime. Assume that $M$ is a compact lightlike hypersurface immersed into $\bar{M}$ with $A_{N} \xi=0$; screen bundle $S(T M)$ is integrable and the Ricci tensor of induced connection $\nabla$ is symmetric. If $\mathrm{H}_{2}$ is a positive constant, then $M$ is a totally umbilical lightlike hypersurface of $\bar{M}$.

Proof. Using the Theorem 9 and multiplying the formula (53) by $H_{2}^{\prime}$ we have

$$
\int_{M}\left(f^{\prime}(h) H_{2}-\langle K, \xi\rangle H_{1} H_{2}\right) d V=0
$$

When $r=2$, we obtain that the Minkowski formula (53) is

$$
\int_{M}\left(f^{\prime}(h) H_{2}-\langle K, \xi\rangle H_{3}\right) d V=0 .
$$

Subtracting the two formulas above we get

$$
\int_{M}\langle K, \xi\rangle\left(H_{1} H_{2}-H_{3}\right) d V=0
$$

Since $H_{2}>0$, from (60) we have $H_{1}^{2} \geq H_{2}>0$, so $H_{1}$ does not vanish on $M$. Since $H_{2}$ is an intrinsic quantity and it does not depend on the chosen orientation, then we can choose an appropriate orientation such that $H_{1}>0$. From the generalization Cauchy-Schwarz inequality in [17] we have $H_{2}^{2}-H_{1} H_{3} \geq 0$; then

$$
H_{1} H_{2}-H_{3} \geq H_{1} H_{2}-\frac{H_{2}^{2}}{H_{1}}=\frac{H_{2}}{H_{1}}\left(H_{1}^{2}-H_{2}\right) \geq 0,
$$

where the equality holds only at the point when $k_{1}^{\prime}=k_{2}^{\prime}=$ $\cdots=k_{n}^{\prime}$. Besides, $\langle K, \xi\rangle>0$, so we have $H_{1} H_{2}-H_{3} \equiv 0$; then it is easy to know that there exists constant $\lambda$ making that $A_{N}=\lambda I$ on screen bundle. Since $A_{N} \xi=0$, then from Corollary 5 we have that $M$ is totally umbilical.
Theorem 12. Let $\bar{M}=-I \times{ }_{f} F^{n+1}$ be a spatially closed $R W$ spacetime. Assume that $M$ is a compact lightlike hypersurface immersed into $\bar{M}$ with $A_{N} \xi=0$; screen bundle $S(T M)$ is integrable and the Ricci tensor of induced connection $\nabla$ is symmetric. If $H_{r}$ and $H_{r-1}$ are both constants $(1 \leq r \leq n)$, then the hypersurface $M$ must be totally umbilical.

Proof. Using Theorem 9 and multiplying the formula (53) by constant $H_{r}$, then we obtain

$$
\int_{M}\left(f^{\prime}(h) H_{r-1} H_{r}+\left\langle\partial_{t}, \xi\right\rangle H_{r}^{\prime 2}\right) d V=0
$$

Multiplying the $r$-th formula by constant $H_{r-1}$, then we have

$$
\int_{M}\left(f^{\prime}(h) H_{r-1} H_{r}-\langle K, \xi\rangle H_{r-1} H_{r+1}\right) d V=0 .
$$

Subtracting the two formulas above we get

$$
\int_{M}\langle K, \xi\rangle\left(H_{r}^{2}-H_{r-1} H_{r+1}\right) d V=0 .
$$

By using the generalization of Cauchy-Schwarz inequality we have

$$
H_{r}^{2}-H_{r-1} H_{r+1} \geq 0,
$$

where the equality holds only at the point $k_{1}=k_{2}=\cdots=k_{n}$. Besides, $\langle K, \xi\rangle>0$, so we have $H_{r}{ }^{2}-H_{r-1} H_{r+1} \equiv 0$; then there exists constant $\lambda$ making that $A_{N}=\lambda I$ on screen bundle. Since $A_{N} \xi=0$; thus by using the Corollary 5 , we have that $M$ is totally umbilical.

Theorem 13. Let $\bar{M}=-I \times{ }_{f} F^{n+1}$ be a spatially closed $R W$ spacetime. Assume that $M$ is a compact lightlike hypersurface immersed into $\bar{M}$ with $A_{N} \xi=0$; screen bundle $S(T M)$ is integrable and the Ricci tensor of induced connection $\nabla$ is symmetric. If $H_{1}>0, \ldots, H_{r}>0$ and $H_{r}$ is constant, where $2 \leq r \leq n-1$, then the hypersurface $M$ is totally umbilical in $\bar{M}$.

Proof. In the same way as the proof of Theorem 11, we have

$$
\int_{M}\langle K, \xi\rangle\left(H_{1} H_{r}-H_{r+1}\right) d V=0,
$$

From the generalization of Cauchy-Schwarz inequality we have for any $1 \leq i \leq n$

$$
H_{i}^{2}-H_{i-1} H_{i+1} \geq 0 \text {, }
$$

where the equality holds only at the point $k_{1}=k_{2}=\cdots=k_{n}$. Since $H_{k}>0,1 \leq k \leq r$, then we have

$$
H_{1} \geq \frac{H_{2}}{H_{1}} \geq \cdots \geq \frac{H_{r}}{H_{r-1}},
$$

where the equality holds only at the point $k_{1}=k_{2}=\cdots=k_{n}$. From $\langle K, \xi\rangle>0$ we have $H_{1} H_{r}-H_{r+1} \equiv 0$; then there exists constant $\lambda$ making that $A_{N}=\lambda I$ on screen bundle. Since $A_{N} \xi=0$, thus by using the Corollary 5 , we have that $M$ is totally umbilical. 


\section{Compact Lightlike Hypersurface with Elliptic Point}

In this section, an elliptic point we mean is a point where all the principal curvature $k_{i}(p)$ of $A_{N}$ restricted on the screen bundle has the same sign. Now we will derive the result about the existence of elliptic point lightlike hypersurface.

Lemma 14. Let $M^{n+1} \rightarrow-I \times{ }_{f} F^{n+1}$ be a compact lightlike hypersurface immersed into a spatially closed RW spacetime, where $A_{N} \xi=0$, screen bundle $S(T M)$ is integrable and the Ricci tensor of induced connection $\nabla$ is symmetric. When $f^{\prime}(h)$ does not vanish on $M$, then (i). If $f^{\prime}(h)>0$ on $M$, then there exists an elliptic point of $M$ with respect to its future-pointing Gauss map. (ii) If $f^{\prime}(h)<0$ on $M$, then there exists an elliptic point of $M$ with respect to its past-pointing Gauss map.

Proof. When $f^{\prime}(h)>0$, let us choose on $M$ the futurepointing Gauss map $T$ and let $p_{0} \in M$ be the point where the height function $h$ attains its minimum on $M$, so we have $\nabla h\left(p_{0}\right)=0, \nabla^{2} \rho\left(p_{0}\right) \geq 0$ and $\left\langle\xi, \partial_{t}\right\rangle>0$. From $\nabla_{X}(\nabla h)=$ $-(\log f)^{\prime}(h)(\langle\nabla h, X\rangle \nabla h+X)-\left\langle\partial_{t}, \xi\right\rangle A_{N} X$ we have

$$
\nabla^{2} h_{p_{0}}\left(e_{i}, e_{i}\right)=-(\log f)^{\prime}(h)\left(p_{0}\right)-\left\langle\partial_{t}, \xi\right\rangle k_{i}\left(p_{0}\right) \geq 0,
$$

where $\left\{e_{1}, \ldots, e_{n}\right\}$ is a basis of principal direction at $p_{0}, i=$ $1, \ldots, n$, so we have

$$
k_{i}\left(p_{0}\right) \leq \frac{1}{\left\langle\partial_{t}, \xi\right\rangle}\left(-(\log f)^{\prime}(h)\left(p_{0}\right)\right)<0
$$

then we obtain the result.

In a similar way, when $f^{\prime}(h)<0$ we choose the pastpointing Gauss map on $M$ such that $\left\langle\partial_{t}, \xi\right\rangle<0$; consider $p_{0} \in M$ to be the point where the height function $h$ attains its maximum on $M$. Now we have $\nabla h\left(p_{0}\right)=0$,

$$
\nabla^{2} h_{p_{0}}\left(e_{i}, e_{i}\right)=-(\log f)^{\prime}(h)\left(p_{0}\right)-\left\langle\partial_{t}, \xi\right\rangle k_{i}\left(p_{0}\right) \leq 0 .
$$

From $f^{\prime}(h)<0$ we have

$$
k_{i}\left(p_{0}\right) \leq-\frac{1}{\left\langle\partial_{t}, \xi\right\rangle}(\log f)^{\prime}(h)\left(p_{0}\right)<0 .
$$

Then we finish the proof.

Theorem 15. Let $\bar{M}=-I \times{ }_{f} F^{n+1}(n \geq 4)$ be a spatially closed RW spacetime. Assume that $M$ is a compact lightlike hypersurface immersed into $\bar{M}$ which contains an elliptic point, $A_{N} \xi=0$; screen bundle $S(T M)$ is integrable and the Ricci tensor of induced connection $\nabla$ is symmetric. If $H_{k}$ is constant, where $3 \leq k \leq n-1$, then the hypersurface $M$ is totally umbilical in $\bar{M}$.

Proof. From Lemma 14 we have that there exists elliptic point $p_{0}$ with respect to the future-pointing Gauss map $T$, such that all the principle curvature of $A_{N}$ restricts on $S(T M)$; then constant $H_{k}=H_{k}\left(p_{0}\right)$ is positive. By using Garding inequality we have

$$
H_{1} \geq H_{2}^{1 / 2} \geq \cdots \geq H_{k-1}^{1 /(k-1)} \geq H_{k}^{1 / k}>0,
$$

where the equality holds only at the point where $k_{1}=$ $k_{2}=\cdots=k_{n}$. From $f^{\prime}(h)>0$ on $M$ we have that $f^{\prime}(h) H_{k} \geq f^{\prime}(h) H_{k}^{(k-1) / k}$. Integrating this inequality and using the Minkowski formula we obtain

$$
\begin{aligned}
H_{k} \int_{M}\langle K, \xi\rangle d V & =\int_{M} f^{\prime}(h) H_{k-1} d V \\
& \geq H_{k}^{(k-1) / k} \int_{M} f^{\prime}(h) d V \\
& =H_{k}^{(k-1) / k} \int_{M}\langle K, \xi\rangle d V .
\end{aligned}
$$

So we have

$$
\int_{M}\left(H_{1}-H_{k}^{1 / k}\right)\langle K, \xi\rangle d V \leq 0
$$

Besides, we already know that $\langle K, \xi\rangle>0$ and $H_{1}-H_{k}^{1 / k} \geq 0$, so we have $H_{1}-H_{k}^{1 / k} \equiv 0$; then $k_{1}=k_{2}=\cdots=k_{n}$; that is, there exists constant $\lambda$ making that $A_{N}=\lambda I$ on screen bundle. Since $A_{N} \xi=0$, thus by using the Corollary 5 we know that $M$ is totally umbilical.

When the hypersurface immersed into the RW spacetime with linearly related $r$-mean curvature, then we have the following totally umbilical result.

Theorem 16. Let $\bar{M}=-I \times{ }_{f} F^{n+1}(n \geq 4)$ be a spatially closed RW spacetime. Assume that $M$ is a compact lightlike hypersurface immersed into $\bar{M}$ with $A_{N} \xi=0$; screen bundle $S(T M)$ is integrable and the Ricci tensor of induced connection $\nabla$ is symmetric. If $f^{\prime}(h) \neq 0$, there are exists integers $k$ and $s$, where $0 \leq r<s<n$ or $0<r<s \leq n$ and the higher-order mean curvature restricted on $S(T M)$ is linearly related by

$$
H_{s}=a_{k} H_{k}+a_{k+1} H_{k+1}+\cdots+a_{s} H_{s}
$$

where $a_{k}, \ldots, a_{s}$ are nonnegative; then the hypersurface $M$ is totally umbilical.

Proof. (i) When

$$
0 \leq r<s<n,
$$

from the formula (79) and the $s+1$ th Minkowski formula, we have

$$
\begin{aligned}
\int_{M}\langle K, \xi\rangle H_{s+1} d V & =\int_{M} f^{\prime}(h) H_{s} d V=\sum_{i=r}^{s-1} a_{i} \int_{M} f^{\prime}(h) H_{i} d V \\
& =\sum_{i=r}^{s-1} a_{i} \int_{M}\langle K, \xi\rangle H_{i+1} d V
\end{aligned}
$$

then we have

$$
\int_{M}\langle K, \xi\rangle\left(H_{s+1}-\sum_{i=r}^{s-1} a_{i} H_{i+1}\right) d V=0 .
$$


Since $f^{\prime}(h) \neq 0$, we suppose that $f^{\prime}(h)$ is positive on $M$; then from Lemma 14 we know that there exists a point $p_{0} \in M$ where all the principal curvature of $A_{N}$ restricted on $S(T M)$ is negative. Denote $M_{0}$ as the connected component of $\{p \in$ $\left.M: H_{s}(p)>0\right\}$ containing the elliptic point $p_{0}$, so $M_{0}$ is a nonempty subset of $M$. If $M_{0}$ is also closed, then $M_{0}=M$; now we will prove that it is closed. From $H_{s}\left(p_{0}\right)>0$ we have that there exists at least one positive coefficient $a_{k}$, such that $a_{k}>0$; then by using Garding inequalities we have for any point $p \in M_{0}$,

$$
H_{j}^{s / j} \geq H_{s}(p)>0
$$

where $1 \leq j \leq s-1, a_{j} \geq 0$; then we obtain $H_{s}(p) \geq a_{k} H_{k}(p)$.

If $k=0$, then $H_{s} \geq a_{0}>0$ on $M_{0}$, so $M_{0}$ is closed.

If $k \geq 1$, then on $M_{0}$ we have $H_{k}^{s / k} \geq H_{s} \geq a_{k} H_{k}>0$, so $H_{k}^{(s-k) / k} \geq a_{k}$ on $M_{0}$; that is, $H_{s} \geq a_{k} a_{k}^{k /(s-k)}=a_{k}^{s /(s-k)}>0$; then $M_{0}$ also is closed.

Thus $M_{0}=M$ and (82) always holds on $M$.

From formula (83) and Cauchy-Schwarz inequality we have $H_{j}>0$ and

$$
H_{j}^{2}-H_{j-1} H_{j+1} \geq 0,
$$

where the equality holds only at the point $k_{1}=k_{2}=\cdots=k_{n}$, $1 \leq j \leq s$. Then it equals

$$
\frac{H_{s+1}}{H_{s}} \leq \frac{H_{s}}{H_{s-1}} \leq \cdots \leq \frac{H_{j+1}}{H_{j}} \leq \cdots \leq \frac{H_{2}}{H_{1}} \leq H_{1},
$$

where the equality holds only at the point $k_{1}=k_{2}=\cdots=k_{n}$, $1 \leq j \leq s$.

From (79) and (85) we get that

$$
\frac{H_{s+1}}{H_{s}} \leq \frac{H_{s}}{H_{s-1}}=\sum_{j=r}^{s-1} a_{j} \frac{H_{j}}{H_{s-1}} \leq \sum_{j=r}^{s-1} a_{j} \frac{H_{j+1}}{H_{s}} ;
$$

then we obtain $H_{s+1} \leq \sum_{j=r}^{s-1} a_{j} H_{j+1}$. By using $\langle K, \xi\rangle>0$ and (82) we have

$$
H_{s+1}=\sum_{j=r}^{s-1} a_{j} H_{j+1}
$$

It means that $k_{1}=k_{2}=\cdots=k_{n}$. Since $A_{N}=0$ then by using Corollary 5 we have that $M$ is totally umbilical.

(ii) When $0<r<s \leq n$, now we just need to prove the case $s=n$ and $r>0$; others are the same with (i); by using the Minkowski formula we have that

$$
\begin{aligned}
\int_{M} f^{\prime}(h) H_{n-1} d V & =\sum_{i=r}^{n-1} a_{j} \int_{M}\langle K, \xi\rangle H_{j} d V \\
& =\sum_{j=r}^{n-1} a_{i} \int_{M} f^{\prime}(h) H_{j-1} d V,
\end{aligned}
$$

so we have

$$
\int_{M} f^{\prime}(h)\left(H_{n-1}-\sum_{j=r}^{n-1} a_{i} H_{j-1}\right) d V=0 .
$$

In a similar way, we assume $f^{\prime}(h)$ is positive on $M$, then there exists a point $p_{0} \in M$ where all the principal curvatures of $A_{N}$ restricted on screen bundle are negative. In the same way we obtain that $M_{n}=\left\{p \in M: H_{n}(p)>0\right\}=M$ and $H_{j}^{n / j}(p) \geq$ $H_{n}(p)>0$ at each point $p \in M$. From Newton inequality we have

$$
\frac{H_{n}}{H_{n-1}} \leq \frac{H_{n-1}}{H_{n-2}} \leq \cdots \leq \frac{H_{j+1}}{H_{j}} \leq \cdots \leq \frac{H_{2}}{H_{1}} \leq H_{1},
$$

where the equality holds only at the points $k_{1}=k_{2}=\cdots=k_{n}$. From (79) we have

$$
\frac{H_{n-1}}{H_{n-2}} \geq \frac{H_{n}}{H_{n-1}}=\sum_{j=r}^{n-1} a_{j} \frac{H_{j}}{H_{n-1}} \geq \sum_{j=r}^{n-1} a_{j} \frac{H_{j-1}}{H_{n-2}} ;
$$

then from (89) we obtain that

$$
H_{n-1} \geq \sum_{j=r}^{n-1} a_{j} H_{j-1}
$$

where the equality holds only at the points $k_{1}=k_{2}=\cdots=k_{n}$. By using Corollary 5 we have that $M$ is totally umbilical.

\section{Conflict of Interests}

The authors declare that there is no conflict of interests regarding the publication of this paper.

\section{Acknowledgment}

This work is supported by NSFC (no. 11371076).

\section{References}

[1] S. Y. Cheng and S. T. Yau, "Maximal space-like hypersurfaces in the Lorentz-Minkowski spaces," Annals of Mathematics, vol. 104, no. 3, pp. 407-419, 1976.

[2] D. Brill and F. Flaherty, "Isolated maximal surfaces in spacetime," Communications in Mathematical Physics, vol. 50, no. 2, pp. 157-165, 1976.

[3] Y. Choquet-Bruhat, "Maximal submanifolds and submanifolds with constant mean extrinsic curvature of a Lorentzian manifold," Annali della Scuola Normale Superiore di Pisa, vol. 3, no. 3, pp. 361-376, 1976.

[4] R. Bartnik, P. T. Chruściel, and N. Ó Murchadha, "On maximal surfaces in asymptotically flat space-times," Communications in Mathematical Physics, vol. 130, no. 1, pp. 95-109, 1990.

[5] C. Gerhardt, " $H$-surfaces in Lorentzian manifolds," Communications in Mathematical Physics, vol. 89, no. 4, pp. 523-553, 1983.

[6] A. J. Goddard, "Some remarks on the existence of spacelike hypersurfaces of constant mean curvature," Mathematical Proceedings of the Cambridge Philosophical Society, vol. 82, no. 3, pp. 489-495, 1977.

[7] H. Rosenberg, "Hypersurfaces of constant curvature in space forms," Bulletin des Sciences Mathématiques, vol. 117, no. 2, pp. 211-239, 1993. 
[8] L. J. Alías, A. Brasil, Jr., and A. G. Colares, "Integral formulae for spacelike hypersurfaces in conformally stationary spacetimes and applications," Proceedings of the Edinburgh Mathematical Society, vol. 46, no. 2, pp. 465-488, 2003.

[9] L. J. Alías and A. G. Colares, "Uniqueness of spacelike hypersurfaces with constant higher order mean curvature in generalized Robertson-Walker spacetimes," Mathematical Proceedings of the Cambridge Philosophical Society, vol. 143, no. 3, pp. 703-729, 2007.

[10] K. L. Duggal and A. Bejancu, Lightlike Submanifolds of SemiRiemannian Manifolds and Applications, vol. 364, Kluwer Academic Publishers, Dordrecht, The Netherlands, 1996.

[11] K. L. Duggal and A. Giménez, "Lightlike hypersurfaces of Lorentzian manifolds with distinguished screen," Journal of Geometry and Physics, vol. 55, no. 1, pp. 107-122, 2005.

[12] S. Montiel, "An integral inequality for compact spacelike hypersurfaces in de Sitter space and applications to the case of constant mean curvature," Indiana University Mathematics Journal, vol. 37, no. 4, pp. 909-917, 1988.

[13] L. J. Alías, A. Romero, and M. Sánchez, "Uniqueness of complete spacelike hypersurfaces of constant mean curvature in generalized Robertson-Walker spacetimes," General Relativity and Gravitation, vol. 27, no. 1, pp. 71-84, 1995.

[14] L. J. Alías, A. Romero, and M. Sánchez, "Spacelike hypersurfaces of constant mean curvature and Calabi-Bernstein type problems," The Tohoku Mathematical Journal, vol. 49, no. 3, pp. 337345, 1997.

[15] C. C. Hsiung, "Some integral formulas for closed hypersurfaces," Mathematica Scandinavica, vol. 2, pp. 286-294, 1954.

[16] I. Bivens, "Integral formulas and hyperspheres in a simply connected space form," Proceedings of the American Mathematical Society, vol. 88, no. 1, pp. 113-118, 1983.

[17] G. Hardy, J. E. Littlewood, and G. Póyla, Inequalities, Cambridge Mathematical Library, 2nd edition, 1989. 


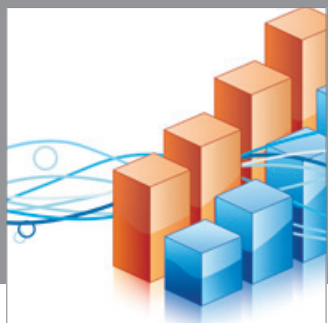

Advances in

Operations Research

mansans

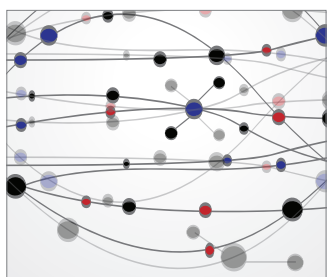

The Scientific World Journal
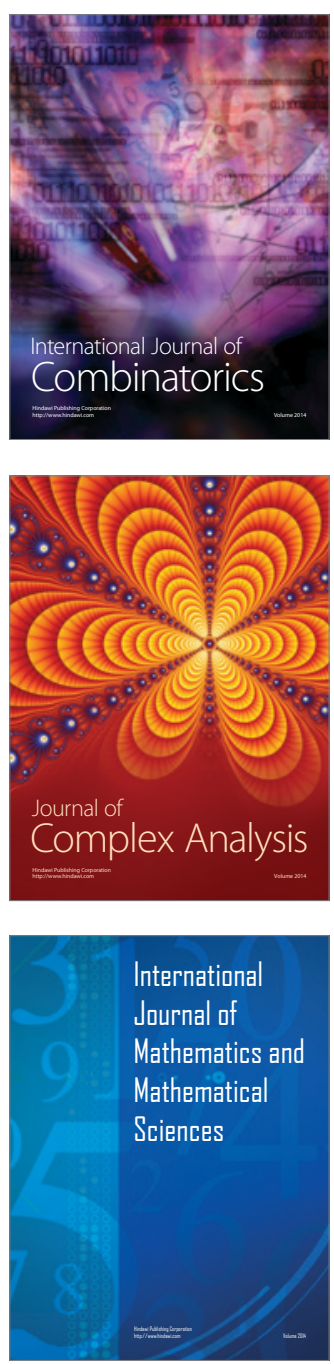
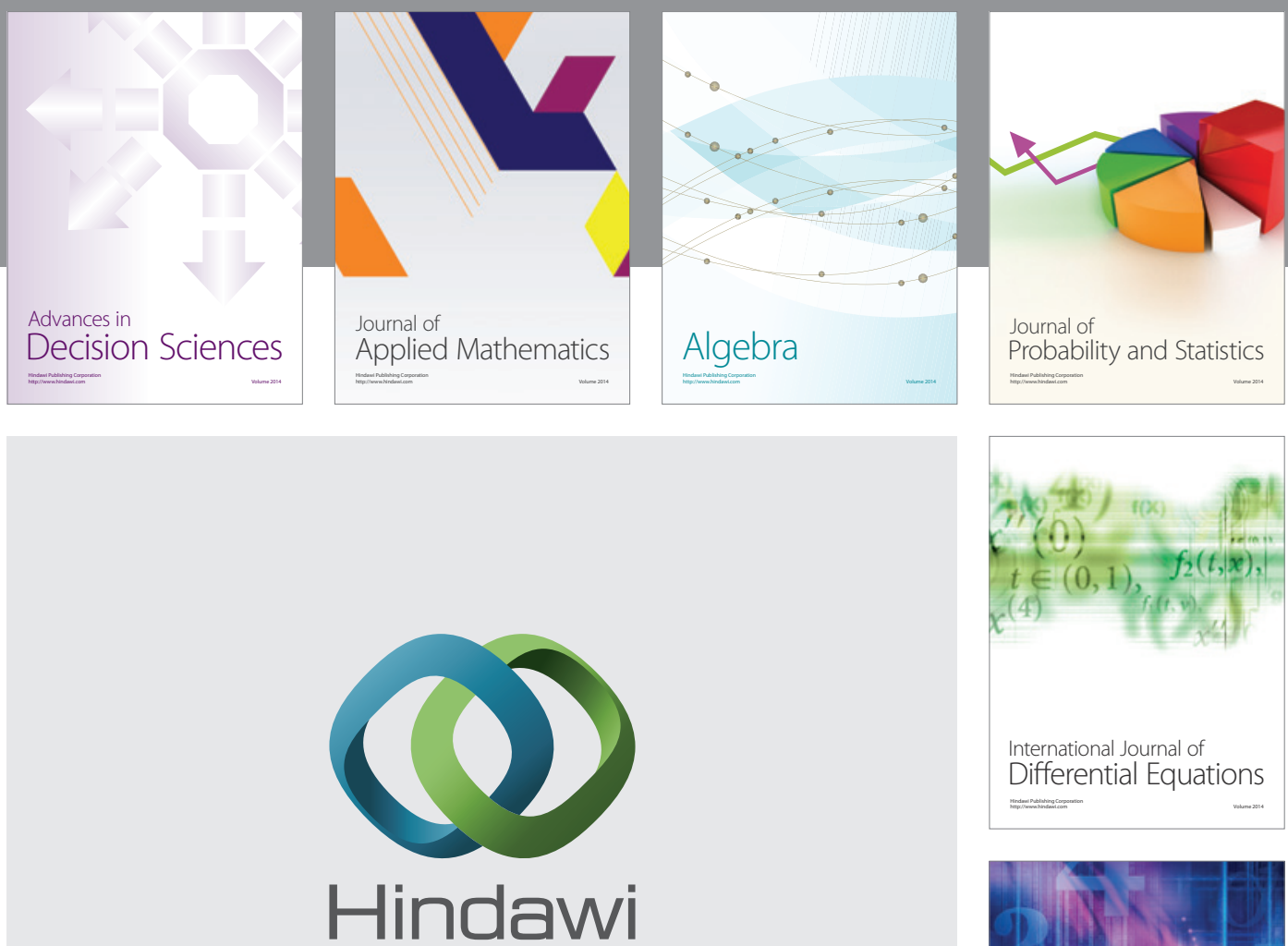

Submit your manuscripts at http://www.hindawi.com
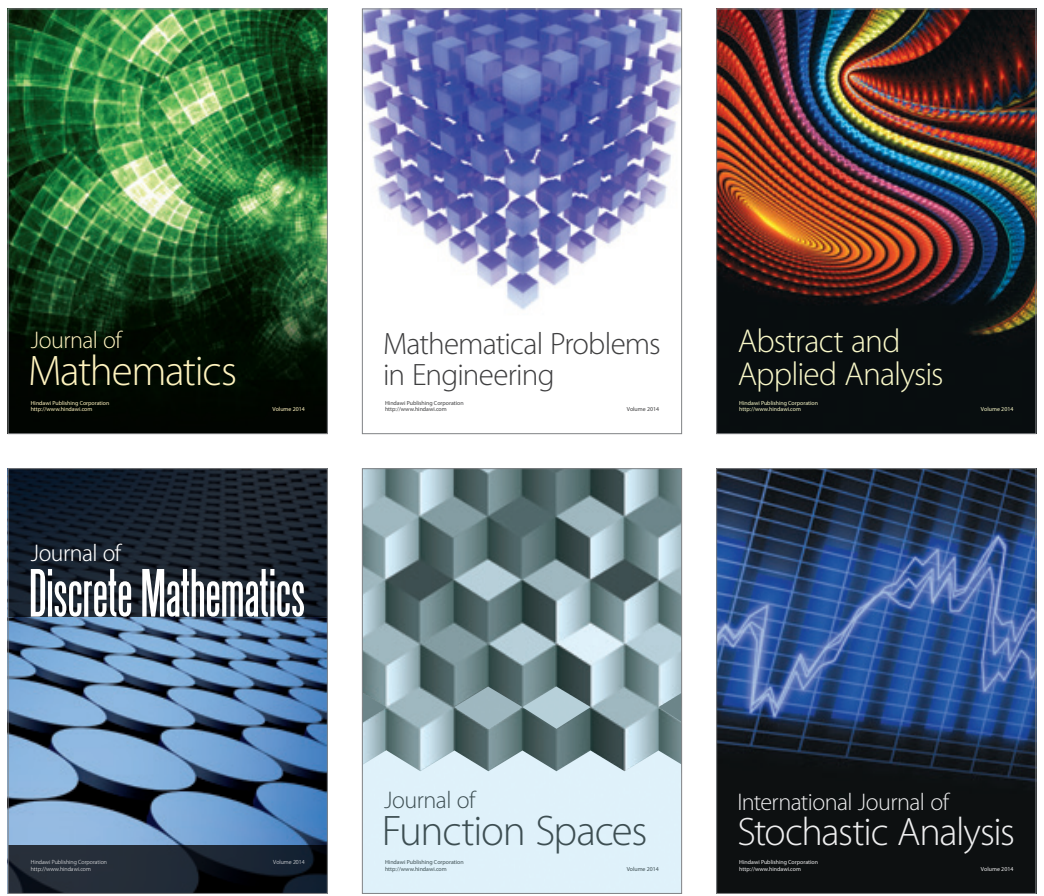

Journal of

Function Spaces

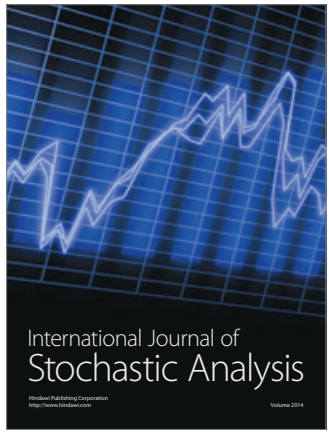

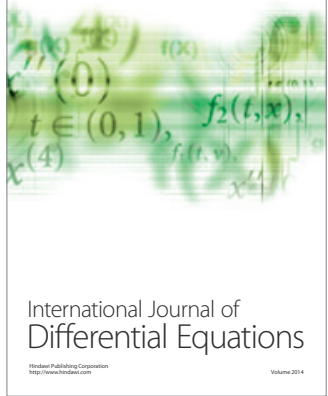
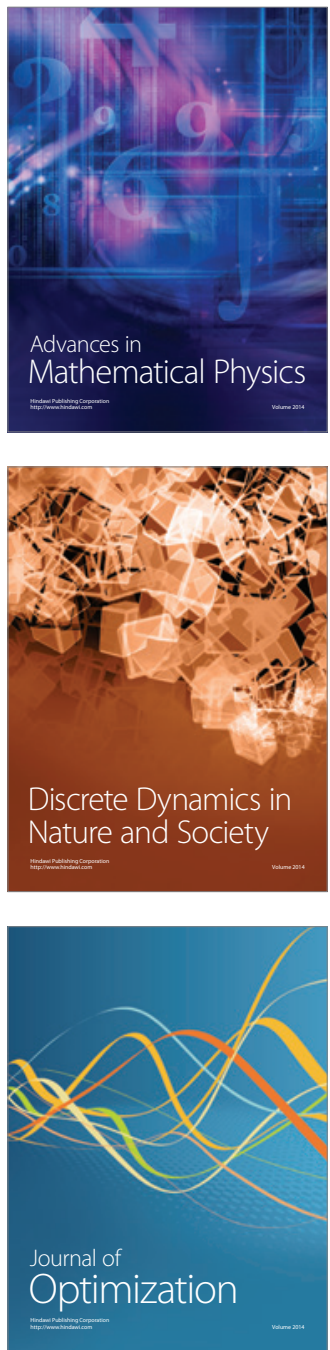